\title{
3 Die griechische Historiographie zwischen Vergangenheit und Gegenwart
}

Meine Überlegungen $z u$ den griechischen Vergangenheitsvorstellungen haben bisher diejenige Gattung weitestgehend ignoriert, die nach allgemeiner Kenntnis als die Referenz zu dieser Thematik angesehen und auch in der gelehrten Praxis entsprechend behandelt wird: die Historiographie. Sie soll nun in den Blick genommen werden, aber vor dem Hintergrund der bisherigen Beobachtungen. ${ }^{1}$ Diese sollen deshalb zunächst kurz resümiert werden. Im Suchen nach der Repräsentation von Geschichte in der griechischen Antike ging ich von den Überlieferungen vergangener oder als vergangen angesehener Ereignisse und Zustände aus, die von den Griechen als gemeinsame Vergangenheit geteilt wurden und die insofern auch für ihr Selbst- und Fremdverständnis und damit für ihre Formierung als distinkte Gruppe, Nation, Volk, Ethnie - kurzum für ihr nation building bzw. ihre Ethnogenese - relevant waren. In Anlehnung an bestimmte konzeptionelle Überlegungen vornehmlich der Kulturanthropologie verwende ich dafür den Begriff intentionale Geschichte.

In den beiden ersten Kapiteln habe ich in diesem Rahmen vor allem herausgearbeitet, dass die intentionale Geschichte der Griechen zunächst vor allem in Mythen greifbar ist, so dass man auch von Mythistorie sprechen könnte. Diese Geschichte ist ursprünglich eingebunden in kosmisches Geschehen, sie rechnet mit der Nähe, ja der unmittelbaren Kommunikation von Göttern und Menschen und bildet eine heroisch-epische Zeit, mit der die jeweils eigene Zeit der Späteren - bei allen durchaus gesehenen Differenzen - durch und durch verbunden ist, auf vielfältige Weise und zum Teil auf verschlungenen Wegen. Für diese Geschichte ist charakteristisch, dass sie ganz wesentlich das Produkt dichterischer bzw. künstlerischer Kreativität und Überlieferung ist. Das gilt es ganz besonders festzuhalten, weil dieser Modus der griechischen Vergangenheitsrepräsentation sozusagen erhalten bleibt. Bei allen Innovationen und Wandlungen, die wir konstatieren können - und gleich werden wir es mit einem massiven Neuansatz zu tun bekommen - bleibt diese ästhetisch-literarische Grundierung eine Art basso continuo.

Innerhalb dieses Rahmens verdienen zwei - ebenso folgenreiche und wirkungsmächtige - Aspekte besondere Beachtung, die Kontexte und Medien sowie die Formen und Inhalte der Mythistorie. Ich habe diese Aspekte vornehmlich im Blick auf ihre ursprüngliche Phase, im wesentlichen den Zeitraum vom 8. bis zum

1 Das folgende Kapitel entspricht in weiten Teilen Gehrke 2014, führt aber einige Aspekte weiter.

(cc)BY-NC-ND (C2014, Hans-Joachim Gehrke.

Dieses Werk ist lizenziert unter der Creative Commons Attribution-NonCommercial-NoDerivatives 3.0 Lizenz. 
6. Jahrhundert, in den ersten beiden Kapiteln getrennt behandelt, obgleich sie eng zusammengehören, ja im Grunde eine Einheit bilden. Deshalb seien sie im Folgenden en bloc noch einmal kurz präsentiert: Die „soziale Oberfläche“ ${ }^{2}$ dieser Mythistorie bestand vor allem in ihrer geradezu unmittelbaren Einbettung in die wesentlichen Formen und Medien der sozialen Kommunikation. Die jeweiligen literarischen Produkte, konkret: die erzählten Mythen, hatten ihren Sitz im Leben in der direkten Darbietung aus geselligen oder kultischen (oder daraus gemischten) Anlässen und kleideten sich in entsprechend rituelle bzw. ritualisierte Formen. Die Dominanz des Performativen äußerte sich in einer engen face-to-faceKommunikation zwischen Autor und Publikum, nicht selten sogar in der Mitwirkung der Nicht-Produzenten als Akteure der Darbietungen, etwa als Sänger und Tänzer.

Diese Phänomene sind nicht nur aus den überlieferten Texten zum Teil zu rekonstruieren, sondern werden dort auch direkt präsentiert und insofern auch thematisiert, ja - wie wir noch sehen werden - auch problematisiert: Schon im 1. Gesang der Odyssee taucht im geselligen Kreise von Penelopes Freiern der Sänger mit dem sprechenden Namen Phemios (so viel wie „Künder“) auf, der mit den schrecklich-schönen Geschichten der Rückkehr der Troiakämpfer sein Publikum „bezaubert“, ${ }^{3}$ nicht anders als am Hofe des Phäakenkönigs der blinde Sänger Demodokos, der lustige Göttergeschichten von sich gibt, aber auch bedrückende und zu Tränen bewegende Lieder vom Kampf um Troia.

Was an den Gesängen besonders bewegt und insofern mit der Aktivität ihrer Produzenten verbunden ist - nicht zuletzt auch bei den von Homer dargestellten Sängern - sind die Leistungen von Helden. Gerade vor dem sozialen Hintergrund wird erkennbar, dass die Orientierung dieser Literatur auf den Nachruhm (kleos) bzw. das Nachleben, die posterity, von wesentlicher Bedeutung ist. Man schaute in die Zukunft und stellte sich vor, dass die Sänger der eigenen Zeit durch ihre Produkte für ein Weiterleben der Leistungen und eben damit für den Ruhm sorgten und dass es auch später Gesänge gegen werde, die das à la longue verbürgten - so wie man auf die Heroen (mit ihren Sängern) zurückblickte, von denen man durch Homer und unzählige andere Poeten erfuhr und die man für historische Gestalten, ja eigene Vorfahren hielt.

Es gab also ein komplexes Beziehungsgeflecht zwischen Vergangenheit, Gegenwart und Zukunft, das man allerdings in seinem Vorstellungshorizont durchaus sortieren konnte. Einerseits lebte man ganz im Gegenwärtigen oder im

2 Zum Begriff s. Luraghi 2001b, 159 mit weiteren Hinweisen (Anm. 54).

3 Hom. Od., 1, 154f., bes. 325-344, bezeichnenderweise im Zusammenhang mit der Verbreitung von kleos (337f.). 
Blick auf die jüngste Vergangenheit, die ja im Vordergrund von Phemios' und Demodokos' Darbietungen stand. Und überhaupt konnte man sich mit den dargestellten Helden und vor allem ihrem Werte- und Motivationshorizont vollkommen identifizieren. Andererseits verstand man das Dargebrachte als Vergangenheit. Es war sogar Teil einer grandiosen und auch zeitlich - nach Generationen strukturierten Vergangenheit, in der schon früh auch ein Plusquamperfekt (in Gestalt des alten Nestor) aufschimmerte. Schließlich konnte man es, nach denselben Prinzipien, also auch genealogisch, bis in die eigene Zeit verlängern bzw. immer weiter fortschreiben.

Das spielte sich nun - abhängig von den jeweiligen Gelegenheiten und den sozialen wie kultischen Reichweiten - an den verschiedensten Orten ab und hatte Relevanz für kleine Dorfgemeinschaften bis hin zu ganzen Regionen oder zur Gemeinschaft der Hellenen, die sich etwa bei den großen Festen in Olympia und Delphi versammelten. Da auch keine dominante Ordnungsmacht existierte, sondern allenfalls die soziale Akzeptanz oder die Regeln der Kunst zählten, herrschte eine unüberschaubare und stets erweiterte bzw. erweiterbare Fülle von Varianten. Manches, besonders solches, das man auf Autoritäten wie Homer oder Hesiod zurückführte, bewahrte man, anderes entdeckte man neu - aus lokalen Überlieferungen - oder man fügte schlicht Neues hinzu, machte es passend gegenüber dem bereits Bekannten oder extrapolierte es aus eigenen Zeiterfahrungen heraus, die man dann nach hinten, in die Vergangenheit projizierte. Wieder gab es eine besondere Verquickung von Vergangenheit und Gegenwart, die auf ähnlich Weise wie zur homerischen Zeit untrennbar waren. Und so füllte sich die griechische Geschichte auch entsprechend der räumlichen Ausdehnung der Griechen bzw. all derer, die sich im Laufe der Zeit dazu rechneten und sich über einen mehr als beachtlichen Raum ausdehnten - was wiederum für die Kraft dieser Geschichten spricht.

Durch und durch trainiert mit dem Satz des Widerspruchs und unseren methodischen Verfahren würden wir hier sofort fragen, wie es denn um die Stimmigkeit dieser Versionen bestellt ist; und wir machen davon bei unseren Analysen des Materials auch regen Gebrauch. Wir müssen aber andererseits auch konstatieren, dass die Griechen durchweg mit der Vielfalt der Varianten sehr gut leben konnten. Das hängt schon mit der Offenheit von Erzählungen selbst zusammen, denen man „inhärente Aushandelbarkeit“ zugeschrieben hat (Bruner 1998, 73). Die Pluralität der Geschichten blieb, gegenüber dem Singular der Geschichte, immer gewahrt. Wir mögen von Konstrukten und Projektionen sprechen, aber das ist die etische Perspektive des modernen Wissenschaftlers. Für die Griechen, jedenfalls für deren Gros, bestand darin ihre Geschichte - im Sinne der schon erwähnten Wissenssoziologie von Peter L. Berger und Thomas Luckmann (o. S. 6) könnten wir davon sprechen, dass das Geflecht der verschiedenen 
Mythen mit ihren Versionen „verdinglicht“ war, also als objektiv, ja geradezu physisch gegeben betrachtet wurde: Man denke etwa nur an die Genealogien. Dass die Griechen diese schillernden Geschichten grosso modo auch als ihre Geschichte ansehen und annehmen konnten, also als insofern „wahre“ Geschichten, verbürgten für sie, jedenfalls in der frühen Zeit, um die es hier noch vornehmlich geht, also vor der partiellen Entzauberung dieser Geschichten, die Musen. Neben dem Wahrheitsanspruch der Sänger und Dichter garantierten sie zugleich aber auch die Eleganz und den ästhetischen Reiz dessen, was jene zu bieten hatten. ${ }^{4}$

Gerade in diesem Zusammenhang tauchte jedoch ein fundamentales Problem auf, das - im Unterschied zu dem durchaus vergleichbaren Problem der Verquickung von Vergangenheit und Gegenwart - bereits in der damaligen Zeit, man könnte fast sagen: von Anfang an, thematisiert wurde, gerade in der poetischen Form; und man kann sich denken, dass es ein Reflex auf die erwähnte Vielfalt und Unbeständigkeit der Varianten und Versionen war, mindestens bei den Fachleuten: Die Kunde der Musen war nicht immer eindeutig, sie konnte in Frage gestellt werden bzw. sie konnte sich gleichsam selbst in Frage stellen (wenn wir das poetologisch verstehen wollen, was da die Sänger und Künstler vorbrachten). Was man auch von den Göttern insgesamt annahm, nämlich dass sie lügen und trügen konnten (oder wenigstens aufs Glatteis führen) und dass die Menschen sich ihrer nicht wirklich sicher sein konnten, ${ }^{5}$ galt auch für die Musen.

Vor kurzem hat François Hartog (2003/2012, 77-83) - bezeichnenderweise gerade am Beispiel der Demodokos-Episode - diesen Aspekt besonders hervorgehoben. Odysseus sieht, so Hartog, in den Musen oder in Apollon die ,Informanten“ des Aoiden, sagt diesem jedoch, er habe „zu perfekt“ (liēn kata kosmon) gesungen, so als sei er persönlich bei den Ereignissen dabei gewesen (hōs pareōn) oder als habe er es von einem anderen (also einem Augenzeugen) gehört (akousas). ${ }^{6}$ Für den Hörer bzw. Konsumenten Odysseus ist das dennoch stimmig (was übrigens bei Hartog etwas zu kurz kommt), denn dieser begründet (gar) gerade das Perfekte und nur durch Augenzeugenschaft zu Erklärende, indem er es auf die Musen oder Apollon als Urheber zurückführt.

Aber mochte sich auch das Publikum beruhigen, die Dichter selbst haben die hiermit aufgeworfene Problematik ins Auge gefasst. Sie haben die Frage nach der Wahrheit sogar ganz gezielt thematisiert und literarisch-poetisch durchdekliniert,

\footnotetext{
4 Primavesi 2009, bes. 106-111.

5 Hierzu s. besonders Deichgräber 1952/1984.

6 Hom. Od. 8, 487-491. Die folgenden Ausführungen verdanken viel einem Vortrag von Bernhard Zimmermann im Wintersemester 2012/13 im Rahmen einer Ringvorlesung des Freiburger Graduiertenkollegs „Faktuales und fiktionales Erzählen“.
} 
übrigens auch sozusagen mit der Gegenprobe: Die Trugreden des Odysseus changieren auf ganz eigene Art zwischen Erfindung bzw. Lüge oder mindestens Camouflage und Wahrheit, so dass Uvo Hölscher sagen konnte, der OdysseeDichter habe „die Qualitäten von Wahrheit und Lüge merkwürdig vertauscht: das Erlogene hat den Schein der Wirklichkeit, während das Wahre das Phantastische ist“. ${ }^{7}$ Selbst seiner treuen Frau gegenüber „reihte“ Odysseus „viel Trügerisches, dem Richtigen ähnlich (pseudea etymoisin homoia), aneinander.“8

Dem Listenreichen mochte man das noch durchgehen lassen. Das Problematische bestand aber eben darin, dass es die Musen selber auch so machten. Schon in dem Anruf, den sie an Hesiod richten, den sie als Hirten durchaus verachtungsvoll begrüßen, sagen sie das selber, mit denselben Worten sogar, die für den trügerischen Odysseus gebraucht werden: „Wir wissen viel Trügerisches, dem Richtigen ähnlich (pseudea etymoisin homoia), zu reden, wir wissen aber auch, wenn wir wollen, Wahres (alēthea) zu verkünden.“9 „Wenn wir wollen“: schöne Garanten der Wahrheit! Und die Sänger und Dichter, die um das Bezirzende und Berückende ihres Tuns wussten und um dessen Spiel zwischen Trug und Wahrheit: schöne Garanten des Nachruhms!

Wenn man wollte, konnte man da schon ins Grübeln kommen. Das haben die Griechen bzw. wenigstens einige von ihnen auch weidlich gemacht; und das soll in diesem zweiten Teil auch besonders beleuchtet werden. Zunächst führte dieses Grübeln zu einer wesentlichen Veränderung, besser: Erweiterung im griechischen Vergangenheitsverständnis und seiner Artikulation. Und die soll im unmittelbar Folgenden im Zentrum stehen. Es geht dabei um nicht mehr und nicht weniger als die Entstehung und die Charakteristik der Geschichtsschreibung als eines besonderen Genres, in dessen Tradition auch wir selber uns nicht selten stellen. In dieser sehen wir ja fast selbstverständlich das Medium oder wenigstens ein zentrales Element der griechischen Vergangenheitspflege, des kollektiven Gedächtnisses der Griechen. Dabei ist sie doch eher etwas Exzeptionelles. Ihre Rolle ist gerade angesichts auch anthropologisch erfassbarer Formen kollektiver Erinnerung nicht selbstverständlich und stellt angesichts der bisher herausgestellten Formen griechischer Mythistorie ein zutiefst erklärungsbedürftiges Phänomen dar.

Der Ausgangspunkt ist klar: Die Griechen hatten einen Schatz von Geschichten, in denen sie durchaus verbindlich - oder auch offen - ihre Vergangenheit erkennen - oder auch fortschreiben - konnten. In diesen waren Gegenwart und

7 Hölscher 1989, 213, vgl. Rengakos in: Rengakos/Zimmermann 2011, 129, der die Stelle zitiert.

8 Homer, Odyssee 19, 203.

9 Hesiod, Theogonie 27f., vgl. hierzu besonders Kannicht 1980; Pratt 1993 (gemäß Hinweisen bei Figal 2000, 301 Anm. 3); zu solcher Ambivalenz in literaturwissenschaftlicher Perspektive vgl. Bruner 1998, 61-63. 
Vergangenheit, „Dichtung und Wahrheit“, Falsches und Richtiges auf vielfältigste Weise verquickt. Dass dies offenkundig nur wenige (diese freilich, wie wir sehen werden, zum Teil nachhaltig) gestört hat, müssen wir konstatieren. Als die Geschichtsschreibung einsetzte, hatten die Griechen jedenfalls längst ihre eigene Vergangenheit(en), in allen Facetten und in Gestalt eines wohl klingenden, gut komponierten und schön aussehenden Ensembles, das im übrigen bestens bekannt war, weil es bestens gepflegt wurde. Sie, die Griechen, haben eben nicht sehnsüchtig auf die Historiographie gewartet, um eine Vergangenheit geschenkt $\mathrm{zu}$ bekommen, und sie haben sie dann auch, als es sie denn gab, weitgehend ignoriert - in dem Maße wahrscheinlich, in dem die heutige Bevölkerung (nicht nur unseres Landes) in ihrer Mehrheit die Debatten und Erkenntnisse der Geschichtswissenschaft nicht zur Kenntnis nimmt.

Von ihrem Ursprung her gehört die Geschichtsschreibung nun einerseits ganz gewiss in den Kontext der eben erwähnten Modi und Gattungen der Erinnerung. Das kann man schon daran erkennen, dass diese in ihrer Zielsetzung (des Tradierens und damit der Nachruhmpflege), mit ihren sprachlichen Fügungen, ihrer verbalen Kunst und ihrer narrativen Struktur massiv auf die Geschichtsschreibung eingewirkt haben. ${ }^{10}$ Wichtige Elemente dieser Erinnerung, ${ }^{11}$ die Genealogien sowie insbesondere die Verquickung von Gründungsgeschichten, Helden, Gründerheroen, Gruppen und Gegenwart ließen sich auch in Prosa ausdrücken. ${ }^{12}$ Wir sprechen hier (im Anschluss an Felix Jacoby etwa) von den Mythographen und Genealogen und kommen mit ihnen teilweise durchaus ins 6. Jahrhundert zurück, auch wenn wir das Gros im 5. Jahrhundert anzusetzen haben. Auf diese Weise kamen die mythistorischen Überlieferungen auch auf das Feld der sich gerade in dieser Zeit immer weiter verbreitenden Prosaliteratur und damit auch einer sich sukzessive professionalisierenden Rhetorik. Zugleich blieben sie Teil der Poesie, wo sich geradezu die Linie einer Entwicklung des historischen Erzählens durch die verschiedenen Genres, ausgehend vom Epos und der folgenden Erschließung anderer Gattungen bis hin zu Dithyrambus und Tragödie abzeichnet. Gerade mit diesen letztgenannten Gattungen und der erwähnten Rheto-

10 Hierzu sind besonders instruktiv Boedeker 1996 und Rengakos 2006, die deshalb statt vieler anderer hervorgehoben seien; zu Herodot s. aber beispielsweise bereits Pohlenz 1937 und vgl. u. S. 78 Anm. 36. S. 83 Anm. 57.

11 Dass jedenfalls die panhellenische Epik, also der wesentliche Repräsentant dieser Mythistorie, die „main source“ für die frühe Geschichte der Griechen war, hat deutlich schon Felix Jacoby $(1949,202)$ hervorgehoben.

12 Strabon $(1,2,6)$ hebt hervor, dass die ältesten einschlägigen Prosaautoren (er nennt den ganz legendären Kadmos sowie Pherekydes und interessanterweise Hekataios) eigentlich nur das „Metrum aufgelöst“ hätten, ansonsten aber ganz noch so wie die Dichter verfahren seien. 
rik zeigt sich die besondere und anhaltende Bedeutung der Performanz, die sogar wegen der Wirkung in die gesellschaftlich-bürgerliche Breite noch gesteigert bzw. erweitert wurde. $^{13}$

Ein neues, ja ganz neues Element kam jedoch demgegenüber durch die im 6. Jahrhundert zunächst in Ionien aufblühende Philosophie und historiē ins Spiel, die ursprünglich mit Erinnerung und Geschichte nichts zu tun hatte. ${ }^{14}$ Es ging dort bekanntlich primär um das Suchen und Fragen nach den archai im Blick auf die Natur, um das Messen und Zählen, um Konstruieren und Harmonisieren, um das kritische Prüfen entlang den Regeln der Rationalität wie der - reflektierenden Subjektivität. Welche Rolle dabei die Frage nach den Göttern spielte und das Unbehagen über deren bisherige Darstellung, nicht zuletzt aber auch das Problem der Wahrheit und Gewissheit gegenüber dem Schein und dem Meinen, zeigen besonders die Fragmente des Xenophanes von Kolophon (ca. 565-470 v. Chr.). ${ }^{15}$ In diesem Milieu ging es auch um das Trügerische der Poetik, um den Riss, der sich von Anfang an durch die uns fassbare Mythistorie hindurchzieht, und hier begnügte man sich nicht mit den Geschichten der Sänger, sondern begab sich auf den Weg „der historiē, die im Sehen, Fragen nach Gesehenem und im Räsonnement zu immer neuen Entdeckungen drängt“ (Deichgräber 1958, 655). Der geistbegabte Kopf, der sich im Sinne eines Geistessportes mit Fragen beschäftigte, die an sich nicht zweckgebundenen waren, bildete nicht erst in der späteren Stilisierung (man denke an Thales und das Lachen der thrakischen Sklavin) einen ganz bestimmten Typus, der sich gerade von der minder begabten und für derart Höheres uninteressierten Masse abhob. Er war entschieden elitär.

Der erste Satz im Werk dessen, der sich mit den skizzierten Anliegen und Regularien auf Geographie und Geschichte, und damit auf das Feld der Erinnerung begab, bringt das ganz entschieden zum Ausdruck: „Hekataios von Milet erzählt Folgendes (hōde mytheitai): Ich schreibe dies, wie es mir wahr zu sein

13 In diese Richtung gehen die Beobachtungen und Überlegungen von Grethlein 2010, s. hierzu vor allem aber auch u. Kap. 4.

14 Diesen Zusammenhängen hat vor allem von Fritz 1967 nachgespürt - ein heute zu Unrecht nahezu vergessenes Werk. Man mag aber auch schon an die Unterscheidung denken, die Polybios $(9,1,4)$ zwischen der älteren Historiographie (Genealogien und Ktiseis) und der politischen Geschichtsschreibung trifft und die in gewisser Weise diese Ausdifferenzierung (wenn auch unter anderen Gesichtspunkten) reflektiert. - Zu der grundsätzlichen Infragestellung der Mythen durch die Philosophie und zur Rolle des elenchos dabei s. die erhellenden Bemerkungen von Figal 2000, 309.314. Vor diesem Hintergrund konnten allerdings Mythen und Dichtungen auch einer Sache adäquat sein, als „schöne Täuschung“ (ebd. 309). Darauf wird am Ende noch zurückzukommen sein.

15 Besonders 21 B 11-16. 18. 23-26. 34-36 Diels/Kranz. Es geht dabei auch um die Kritik daran, dass die homerisch-hesiodeischen Götter Betrüger sind (B 11f.). 
scheint, denn die Reden (logoi) der Griechen sind zahlreich und lächerlich (polloi kai geloioi)“. ${ }^{16}$ Gerade hier sollte mithin kein Platz für die erwähnten traditionellen Formen sein. Es handelte sich, mindestens dem Anspruch nach, um eine klare und gezielte Absage an die herkömmliche Erinnerungspflege und intentionale Geschichte der Griechen und um das Versprechen von etwas Besserem, gerade wenn es um Wahrheit ging, die doch den alten Sängern durch die Musen verbürgt schien, nicht durch ihr eigenes Urteil. ${ }^{17}$ Und das Ringen um die Wahrheit, gerade in ihrer Diskrepanz zu den Meinungen und Ansichten, dem Schein und dem Äußerlichen, stand deutlich im Zentrum der seinerzeitigen Debatten. ${ }^{18}$

In der philosophischen Debatte hat das zu weitreichenden Überlegungen und Konzepten geführt, und noch heute kann man über Wahrheit und ihren Begriff auf verschiedenen Ebenen trefflich streiten. Hier, im Blick auf das Historische und das Erzählte, ging es in einem ganz elementaren Sinne darum, ob dieses, wie es erzählt wurde, mit den erzählten Tatsachen übereinstimmte, ob das, was da berichtet wurde, wirklich und wirklich so passiert war. Das steckt auch in der Definition von Sextus Empiricus (adversus Mathematicos 1, 263), historia sei „die Darlegung von etwas Wahrem und Geschehenem“. ${ }^{19}$ Der analoge Fall ist hier die Ermittlung von wirklich geschehenen Tatsachen vor Gericht, wo die Wahrheit von Aussagen danach bestimmt wird, ob sich das, was ausgesagt wird, so zugetragen hat oder nicht. ${ }^{20}$ Mit anderen Worten, Wahrheit bezieht sich hier nicht auf Kohärenz und Schlüssigkeit oder gar auf Formen von höherer Einsicht, sondern auf die Korrespondenz und Entsprechung zwischen Aussage und empirischem Sachverhalt. Insofern ergab sich im Laufe der Zeit letztendlich eine Differenz zwischen Philosophie, insbesondere Logik und Metaphysik, und Geschichte bzw.

16 Hekataios, FGrH 1 F 1 (vgl. auch o. S. 15f.); zu den gedanklichen Hintergründen und Verfahren s. sehr plastisch Corcella 2006, 40-42 (auch mit Verweis auf Heraklit). Zu Hekataios' Bedeutung am Beginn dieser neuen Richtung s. Bertelli 2001, skeptisch Nicolai 1997. 2007, 17. Zur Rolle des Xanthos (besonders in Relation zu Herodot) vgl. die wichtigen Bemerkungen bei Schepens 2007, 46 und besonders von Fritz 1967 II 348-377.

17 Für die Perseveranz dieses methodischen Ansatzes im griechischen historiographischen Diskurs vgl. Plutarch, Theseus 1, 5 und vgl. vor allem das 4. Kapitel.

18 Hierzu s. die Hinweise bei Gehrke 1993, 11f.

19 Das Beispiel, das Sextus Empiricus direkt anschließend für das Wahre anführt, nämlich die Ermordung Alexanders des Großen durch Gift, zeigt, wie schwer die Ermittlung der Wahrheit im Sinne des „wirklich so“ im einzelnen ist; aber das hat auch schon Thukydides gewusst (vgl. Gehrke, 1993, 9ff.). Zu diesem Aspekt von Wahrheit und Geschichte vgl. auch Walbank 1960 (1985/2011), 401 mit weiteren Belegen aus der rhetorischen Literatur.

20 Dissoi logoi (90 Diels/Kranz) 4, 2 : „Wenn eine Aussage (logos) gemacht wird und wenn es so, wie die Aussage lautet, geschehen ist, dann ist die Aussage wahr; wenn es aber nicht geschehen ist, ist dieselbe Aussage falsch (pseudēs)“, vgl. 4,7. 
Historiographie. In statu nascendi jedoch gehören beide noch eng zusammen, gerade in der Emphase der Wahrheitssuche.

$\mathrm{Zu}$ dem erwähnten elitären Habitus gehörte nun ganz entschieden auch der geistige Konkurrenzkampf mit den anderen (wenigen) Größen, die man jeweils auch, wie das Aristokraten gerne tun, in die Nähe der (vielen) Dummen rückte, also primär ebenfalls der Lächerlichkeit zieh. Gerade bei der erwähnten Wahrheitssuche herrschte der Agon der Debatte, sprachlich und gedanklich in der Nähe des Rechtsstreits: Es ging um das rationale Kontrollieren der gegnerischen Position, den elenchos, im Idealfall bzw. in letzter Konsequenz um deren Widerlegung. Parmenides, einer der radikalsten Denker jener Zeit, in etwa ein Zeitgenosse des Hekataios, empfahl eindringlich (durch den Mund der von ihm evozierten Göttin), „durch Denken (logos) den streitbaren elenchos zu beurteilen (krinein - man beachte dieses auch in der Rechtssprache gebräuchliche Wort)“. ${ }^{21}$ Und hier galt übrigens ganz konsequent der Satz des Widerspruchs. Parmenides’ Schüler Zenon wird eine „irgendwie elenktische und durch Widerrede (antilogiai) <den Gegner> in der Ausweglosigkeit (aporia) einschließende Haltung“ zugeschrieben. ${ }^{22}$

Dieses Procedere blieb nun aber keineswegs auf die unmittelbare Diskussion beschränkt und auf einer insofern lediglich performativen Ebene. Das Medium der Schrift, das auch in der erwähnten Poesie wichtig war, freilich hinter der Darbietung zurücktrat, bekam in der philosophisch-intellektuellen Auseinandersetzung eine ganz spezifische Funktion: Was richtig und wahr war, handelte man nicht nur mündlich aus. Vielmehr verbreitete man seine jeweiligen Positionen auch in Büchern. Die Debatten der Weisen erhielten auf diese Weise eine diachrone Perspektive. Die Verbindungen sind bis heute nicht abgerissen. Wie sich daraus lange Linien des argumentativen Hin und Her ergeben konnten, die man auch bei entsprechender Akzentuierung als eine Geschichte des Fortschritts oder doch wenigstens des Fortschreitens wahrnehmen konnte, zeigt bereits ein Blick auf das erste Buch der aristotelischen Metaphysik, in dem der große Philosoph

2128 B 7,5f. Diels/Kranz. Hier meint elenchos wohl nicht nur die Prüfung (Übersetzung von Hermann Diels: „mit dem Denken bring zur Entscheidung die streitreiche Prüfung, die von mir genannt wurde“), sondern die bereits erfolgte Widerlegung, so die Übersetzung von Fränkel 1962, 405 (von dem ich auch die treffliche Wiedergabe von polydēris mit „streitbar“ gerne übernehme): „,beurteile mit dem Denken die streitbare Widerlegung, wie ich sie gegeben habe“; vgl. Hölscher 1986 („die hart bestreitbare Widerlegung, die von mir vorgebracht worden ist“) und Mansfeld/ Primavesi 2011, („die streitbare Widerlegung, die ich ausgesprochen habe“). Den Bezug von elenchos auch auf das Gerichtswesen bestätigt Plat. Phaidr. 266e/267a; zum elenchos in der Philosophie s. auch Figal 2000, 314. Letztlich wird der Begriff erweitert (bei Sokrates wird das klar) zu einer kontrollierenden Prüfung von Aussagen und Sachverhalten.

22 Plut. Perikles 4,5 = 29 A 4 Diels/Kranz. 
gleichsam die diesbezüglichen Grundsätze und Lehren seiner Vorgänger in ihrer Abfolge skizziert, durchaus eine Art Philosophiegeschichte. ${ }^{23}$

Ähnliches ließe sich auch für andere Felder und Genres, nicht zuletzt die Historiographie, zeigen. Hier geht es immer wieder um ein argumentatives und polemisches Streiten, aber doch damit zugleich immer wieder um ein Wiederaufgreifen, um ein neues und erneutes Aufgreifen von Dingen, die von den Vorgängern behandelt waren und in denen man sich als besser erweisen konnte als diese; und das geschah auf lange zeitliche Distanz (wenn man so will, reicht sie bis heute). Im Blick auch auf solche Zusammenhänge hat Jan Assmann von „Hypolepse“ gesprochen. $^{24}$ Zentral ist jedenfalls auch bei den Historikern der gerade genannte Aspekt des elenchos, des fortlaufenden kritischen Prüfens und ggf. (und nicht selten) Widerlegens, in dem sich die ganze Agonalität der griechischen Intellektuellen und damit der Geschichtsschreiber entfalten konnte und gelegentlich rücksichtslos entfaltet hat.

Am Anfang blieb vieles noch implizit, war aber hinreichend deutlich, so etwa Herodots Bemerkungen zu Hekataios' eigenen Genealogisierungen im Verhältnis zu den großen chronologischen Leistungen der Ägypter; und indem sich Herodot über Autoren der seinerzeitige Kartographie, der gēs periodoi, lächerlich macht, spielt er auf diesen an, denn der hatte ein solches Werk erstellt. In gewisser Hinsicht wird Hekataios auch in die Nähe der von ihm selbst problematisierten logoi gerückt. ${ }^{25}$ Charakteristisch ist noch ein viel späterer Vertreter des Genres, Arrian von Nikomedia (ca. 85/90-150): Er fordert zur Lektüre seiner Vorgänger

23 Aristot. metaph. 1, 3, 983 b 7-1, 10, 993 a 27. Dass dies an den Problemen und Gegenständen orientiert ist und nicht eine Geschichte der Philosophie im engeren Sinne darstellt, macht jetzt Flashar 2013, 213 klar.

24 Assmann 1992, 280-285, vgl. auch o. S. 17; bei Isokr. or. 11, 30 ist das Wort hypolēpsis durchaus im Sinne einer kritisierenden Übernahme gebraucht. - Dafür, dass auf diese Weise, also mit dieser Art von Hypolepse, so etwas wie ein Fortschritt durch innovatives Übertreffen zustande kommt (wie bereits bei den Poeten), s. Corcella 2006, 53; zu diesem Zusammenhang von „convention and innovation“ vgl. generell Marincola 1999, 321. Von unseren Vorstellungen eines wissenschaftlichen Fortschritts sind wir aber durchaus entfernt, vgl. u. S. 113.

25 2,143 (zur Genealogisierung); wo Hekataios als politischer Ratgeber erscheint (5,35. 125f.), ist der Eindruck positiver, weil es hier um einen Rat aus Kennerschaft geht, der zugleich Gelegenheit gibt, Herodots eigenes Wissen in Szene zu setzen (5,35); zu den periodoi 4,36; zu den logoi s.u. Anm. 37; dass er ihn dreimal als logopoios bezeichnet $(2,143,1.5,36.125)$ mag man auch entsprechend auslegen, zumal er auch Äsop so nennt $(2,134,3)$ - Bei Thukydides scheint die Methode eher die eines Verschweigens gewesen zu sein: Hellanikos kritisiert er nur für ein Detail $(1,97,2)$, Herodot kommt gar nicht vor; Bemerkungen im Methodenkapitel, die man häufig als implizite Kritik an Herodot verstanden hatte, muss man wohl anders deuten, s. jetzt Grethlein 2010, 207-209; zur größeren Nähe von Herodot und Thukydides vgl. auch Rogkotis 2006. - Zum „truly polemical spirit“ generell vgl. Schepens 2007, 49. 
auf (an. praef. 3), stellt aber dann seine Leistungen für die griechische Literatur neben die Leistungen Alexanders des Großen für die Weltgeschichte (an. 1, 12, 5).

Gerade mit der kompetitiven Kontrolle kommt die antike Geschichtsschreibung allerdings in ihrer intellektuellen Professionalität in die Nähe der Diskurse der modernen intersubjektiv-kritischen Geschichtswissenschaft zu stehen, die die Pluralität steigert (Le Goff 1988, 193.197f.) und sie zugleich, wegen des Kriteriums der Objektivität im Sinne einer intersubjektiven Nachvollziehbarkeit (ebd. 196), zur „rectification“ führt. ${ }^{26}$ Diese Nähe wird ja gerne und insofern auch nicht zu Unrecht betont, auch wenn das nicht immer reflektiert ist. Sie darf allerdings nicht überbetont werden, wie im Folgenden noch zu zeigen sein wird.

Genau in der Logik dieser im Konkurrenzkampf vorangetriebenen philosophischen Diskurse kam es aber zu dem entscheidenden Sprung in der Genese der Historiographie. Schon längst hatte man in den verschiedenen, älteren und neueren Gattungen der Erinnerungspflege längere Linien in die Vergangenheit gezogen und mittels derer Einmündung in die Gegenwart geschichtlich zu argumentieren gelernt: mit Ansprüchen und Beschuldigungen, wenn es um Konflikt und Konkurrenz, Rechtsstreit oder Krieg ging. Man wusste, wer zuerst im Recht war oder mit dem Ärger angefangen hatte, wer mit wem zusammengearbeitet hatte, wie sich das Geflecht von Aggression und Vergeltung entwickelt hatte. Und zugleich war das angesichts der erwähnten Pluralität immer kontrovers, weil hierzu alle Beteiligten jeweils anderes geltend machen konnten.

Wir können solche Verfahrensweisen noch sehr gut nachverfolgen, und Herodot kannte sie natürlich mindestens ebenso gut wir. Er nimmt sie sogar am Anfang seines Geschichtswerks mit der Frage nach der Ursache des großen und grundsätzlichen Konflikts zwischen Hellenen und Barbaren als Geschichte von Frauenentführungen auf - und geradezu auf die Schippe. ${ }^{27}$ An anderer Stelle, in der von ihm selber so konstruierten Debatte zwischen den Tegeaten und den Athenern um den angemessenen Platz in der Schlachtreihe von Plataiai, konfrontiert er sie mit einem ganz anderen Argumentationsmodell. ${ }^{28}$ Und in beiden Fällen kommt die Gegenwart bzw. die nähere und zugängliche Vergangenheit, wir könnten geradezu sagen: die Zeitgeschichte, ins Spiel. Bereits am Anfang von Herodots Geschichtswerk wird aber auch klar, dass der Rekurs auf die Gegenwart bzw. das Zeitnahe aus einem heuristischen bzw. methodischen Grundproblem resultiert, das genau mit der gerade erwähnten Logik des rational-individuellen Verfahrens zusammen-

26 So Paul Ricoeur (Histoire et vérité, 1955), zitiert bei Le Goff 1988, 199, und Adam Schaff (Histoire et vérité, 1971) ebd. 199f.

27 Herodot 1, 1, 1-1, 5, 3; zu Herodots Proömium generell s. jetzt Węcowski 2004.

28 Herodot 9, 26f., vgl. hierzu Grethlein 2010, 173ff. und zu Herodot generell Giangiulio 2005, mit besonderer Betonung der narratologischen Aspekte. 
hängt, dem sich Herodot verpflichtet weiß. Es geht, genauer gesagt, um die Frage nach der begründeten Erkenntnis, nach dem Wissen, die wegen des subjektiven Faktors auch mit der persönlichen Erfahrung zusammenhängt. Dieses Wissen gründet sich auf die unmittelbare, auf Anschauen beruhende Kenntnis (opsis) sowie auf das persönliche Urteil und die persönliche argumentative Urteilsfähigkeit (gnōmē), die in dem schon von Hekataios bekannten hōs emoi dokeì zum Ausdruck kommt. ${ }^{29}$

Sofern es sich dabei auch um das Ergebnis von Recherchen (historiē) handelt, geht es in einem ganz elementaren Sinne um Zeugenschaft, die des Augen- und Ohrenzeugen. Sie - besonders die Autopsie - wird zum Garanten der Wahrheit: Schon in der Demodokos-Szene macht Odysseus' Bemerkung gegenüber dem Seher genau das deutlich. ${ }^{30}$ Gerade in der intellektuell-kritischen Richtung des griechischen Denkens, von der die Rede war und in die die neue Historiographie hineingehört, wurde die Bedeutung der Augenzeugenschaft hervorgehoben: Wenn Xenophanes von Wissen spricht, so ist dieses auf „Anschauung gegründet“, ${ }^{1}$ und Heraklit bemerkt lapidar, dass die Augen bessere Zeugen als die Ohren seien. Das Zitat kennen wir bezeichnenderweise aus Polybios’ Kritik an dem ,Buchgelehrten' Timaios (12, 27, 1). ${ }^{32}$

Diese markante Betonung der eigenen Anschauung in der Erkenntnistheorie hat für den Umgang mit Geschichte erhebliche Konsequenzen. Letztlich gibt das eigene Wahrnehmen, in Gestalt des Sehens oder doch wenigstens des unmittelbaren Hörensagens (Hdt. 1, 20), den Ausschlag für das Wissen und damit auch das Urteilen. Damit aber verkürzt sich der Blick in die Vergangenheit. Er konzentriert sich maximal auf das Drei- oder Vier-Generationenschema der oral history oder des „kommunikativen Gedächtnisses““.33 Konsequenterweise kam Herodot

29 Herodot 1, 5, 3. - Dafür, dass dieser neue Ansatz „zunächst“ auf Zeitgeschichte verweist, vgl. auch Timpe 1993, 19.

30 Vgl. o. S. 68 und s. besonders Hartog 2003/2012, 78f.; Schepens 2007, 40; weitere Beispiele aus der Epik bei Marincola 1997, $63 f$.

31 Fränkel 1962, 382 zu 21 B 34 Diels/Kranz (mit Anm. 20 für Parallelen); generell s. hierzu Fränkel 1960, 342-349.

32 Herakl. 22 B 101a Diels/Kranz; Walbank stellt hierzu die gnomische Wendung Hdt. 1,8,2 (die Kandaules dem Gyges gegenüber äußert), Menschen vertrauten nun einmal den Ohren weniger als den Augen (weswegen er den Gyges seine schöne Frau nackt sehen lässt, was ihm dann zum Verhängnis wird, wie Herodot unmittelbar vorher schon angekündigt hatte). Hinsichtlich der epistemischen Bedeutung des Sehens könnte man auch an die emphatische Betonung des Sehens für die Erkenntnis am Beginn der aristotelischen Metaphysik (1,1,980 a 21-27) denken. Zur Bedeutung der Autopsie vgl. Reichel 2005, 58 (mit Belegen in Anm. 53) und jetzt grundlegend Schepens 2007.

33 Vansina 1985; Assmann 1992, 48-52; Foxhall 1995, 133ff. Zu den zeitlichen Dimensionen Herodots s. besonders Vannicelli 1993, 13-16, vgl. auch Corcella 2006, 45f. Zur Rolle etwa der 
dann, indem er das nach den o. a. Kriterien möglichst weit zurückverfolgte, bis zu Kroisos. Geschichte in diesem Sinne, nach den Regeln der Philosophie und historiē, von Denken und Gewissheit, ist damit zwangsläufig Zeitgeschichte oder wenigstens so etwas wie Neueste Geschichte. Die hiermit (jedenfalls nach unserem Kenntnisstand) inaugurierte Gattung der Historiographie musste also insofern gar nicht vergegenwärtigen, sie war ganz nah an der Gegenwart, auf Tuchfühlung mit ihr sozusagen. Denn Geschichte war ja überhaupt nur von dieser unmittelbar her $\mathrm{zu}$ erschließen und zu ergründen. Wollte die Historiographie ihren eigenen Ansprüchen treu sein und bleiben, dann musste sie in ihrer Tiefendimension von der Reichweite des Blicks aus der Gegenwart bestimmt sein. Man könnte sich deshalb - und prima facie vor diesem Hintergrund nicht unplausibel - die Frage stellen, ob Herodot überhaupt ein Historiker genannt werden kann.

Das ist freilich bei näherem Zusehen ganz unberechtigt. Herodot weiß sich zwar ganz betont der neuen Methode verpflichtet (und Rosalind Thomas hat bis ins Detail hinein beschrieben, wie weit das ging ${ }^{34}$ ), aber er wirft das Alte nicht über Bord, im Gegenteil. Seine Wendung zu Kroisos nach der Erörterung der Frauengeschichten zu Beginn des Werkes ist insofern keine totale Absage an das spatium mythicum, wie schon vor einiger Zeit Klaus Nickau unterstrichen hat. ${ }^{35}$ Er trägt durchaus die älteren Erinnerungsformen weiter und er hat sich trotz des neuen Ansatzes - wir dürfen durchaus hinzufügen: glücklicherweise - nicht auf das Neue und direkt Ermittelbare beschränkt bzw. er hat den Begriff des Ermittelbaren gerade durch die Dimension des Hörensagens, der logoi eben, ausgedehnt. Denn sein primäres Anliegen war nicht die Recherche an sich, sondern das Bewahren oder umgekehrt: das Verhindern des Vergessens (mē exitēla genētai).

Damit verrät er sich auf jeden Fall und sehr markant als Historiker, und damit steht er zugleich der episch-literarischen Zielsetzung der Nachruhmpflege sehr nahe, wie er ja auch expressis verbis davon spricht, dass die großen Leistungen der Menschen nicht „ohne Ruhm“ (akleā) sein sollten. Somit gehört zum Gegenstand des Forschens auch, was man früher und über Früheres und noch Früheres erzählt hat. Die logoi der Hellenen (und anderer) werden nicht so (generell) verworfen, wie es Hekataios angekündigt hatte. Wenn man so will, handelt es

Großeltern in traditionalen Gesellschaften in diesem Zusammenhang vgl. Connerton 1989, 39 (mit weiteren Hinweisen).

34 Thomas 2000.

35 Nickau 1990, 91-97. Wie dieser größere Vergangenheitsraum organisiert war, hat Vannicelli 2001 dargelegt; vgl. jetzt auch Bichler 2013. 
sich um einen Kompromiss zwischen der alten intentionalen Geschichte und der modernen kritischen Recherche-Geschichte. ${ }^{36}$

Mir scheint, dass Herodot das auch selber verdeutlicht hat: Als Berichterstatter und damit als Erzähler seiner historiē, seiner Erforschung, verzeichnete er auch, was aktuell als Geschichte kursierte, auch logoi, die in ältere Zeiten zurückführen. Für diese freilich übernimmt er keine intellektuelle Garantie, wie er am Beispiel der Diskussionen um die Nichtbeteiligung von Argos am Kampf gegen Xerxes zeigt, in dem es um die mythistorische Verwandtschaft von Argivern und Persern, also um ein traditionelles Argumentieren ging. ${ }^{37}$ Im übrigen nahm Herodot zu der entfernten Vergangenheit durchaus Stellung, zumal wenn es um die zentrale HellenenBarbaren-Thematik ging. ${ }^{38}$ Zudem nimmt er Deutungen der Vergangenheit von der Gegenwart her vor und lässt zugleich Licht von der Vergangenheit auf die Gegenwart fallen, bietet also eine ganz eigene Verschränkung von Vergangenheit und Gegenwart. ${ }^{39}$ Überhaupt hielt er dafür, dass die historischen Ereignisse erst abgeschlossen sein müssten, damit sie ihre didaktische Wirkung entfalten konnten. ${ }^{40}$ Er befand sich also gleichsam in einer mittleren Position. Damit stand er der traditionellen und intentionalen Geschichte nicht völlig fremd gegenüber. Er sucht sie $\mathrm{zu}$ integrieren, wie er sich ja auch sprachlich und narrativ auf ihre Formen bezieht. ${ }^{41}$ Dies erklärt nicht zuletzt auch seine Attraktivität.

Allerdings bildet das kritisch-rationale Verfahren auch bei ihm die erkenntnistheoretische Grundlage. Der entspricht im übrigen auch - das sei hier nur nebenbei gesagt - die Bemühung um eine möglichst präzise Wiedergabe der chronologischen Gegebenheiten wenigstens in dem erwähnten zeitlichen Horizont; hier bildet immer noch Hermann Strasburger den Ausgangspunkt, wie gerade durch neuere Untersuchungen erhärtet wird. ${ }^{42}$ Er nutzt sie auch zur kritischen Prüfung älterer Erzählungen, indem er sie durch die Demonstration chronologischer Unmöglichkeit als unrichtig erweist. ${ }^{43}$ Und ganz Ähnliches lässt sich

36 Vgl. hierzu ähnlich, aber mit je anderen Akzentuierungen Candau Morón/González Ponce/ Chávez Reino 2004; Stadter 2004; Calame 2006; Rutherford 2012 (der Thukydides einschließt); bei Wesselmann 2011 sind die vorgegebenen Erzählstrukturen besonders stark betont. Zahlreiche Einzelbeispiele bietet der Sammelband Giangiulio 2005, vgl. ferner auch Biraschi 1989.

37 Herodot 7, 152. Solche Distanzierung bezieht sich auch auf Hekataios, und zwar auf ein mythistorisches Ereignis, die Vertreibung der Pelasger aus Attika. Der Vorgänger rückt damit übrigens in die Nähe der logoi, deren Problematik er selber herausgestellt hatte, s.o. S. $15 f .72$.

38 Nickau 1990, 91-97.

39 Raaflaub 2002, 21.

40 Grethlein 2009, 215.

41 Vgl. die Hinweise o. S. 70 Anm. 10. 78 Anm. 36 sowie generell jetzt auch Proietti 2012b.

42 Strasburger 1956/1965/1982, vgl. Vannicelli 1993, 13-16; Bichler 2013, 23-26.

43 Ein schönes Beispiel 2,134,1 (ouk orthōs legontes). 
über die räumliche Komponente sagen: Herodot war vertraut mit der neuen Weltsicht der ionischen Geographie und bezieht diese in seinen elenchos mit ein. ${ }^{44}$ Darüber hinaus ging es ihm auch und gerade darum, mit seinen Recherchen in der Vergangenheit auf die aktuelle Politik zu wirken und deren Verständnis und Beurteilung zu fördern - auch dies im Sinne von ratio und Überlegung.

Generell beobachtet man (neben den erwähnten chronologischen Begründungen) ganz bestimmte Argumentationsstrukturen, wenn es um die Ermittlung älteren Geschehens geht, das nicht mehr der opsis, sondern nur noch (aber doch wenigstens) durch Erzählungen, logoi eben, der akoē zugänglich ist. ${ }^{45}$ Die Diskussion um ein famoses Ereignis der spätarchaischen Geschichte, die Zerstörung des sprichwörtlich reichen Sybaris durch Kroton und deren Begleitumstände, wurde intensiv geführt. Dabei argumentierte man - seitens der Beteiligten und ihrer Nachfahren, aber in einem auch von Herodot geteilten Rahmen - u.a. mit manifesten und noch greifbaren Monumenten und noch existenten und wirksamen Kulttraditionen, die man als Beweise bzw. Indizien anführt. In die Gegenwart reichende Relikte im weitesten Sinne, in diesem Falle vor allem noch auffindbare Merkposten im Gelände (ohnehin, wie wir sahen, in der intentionalen Geschichte häufig markiert), spielen also eine besondere Rolle.

Daneben sind hypothetische Erwägungen über einen gedachten anderen Verlauf der Ereignisse wichtig, die eine andere als die definitiv eingetretene aktuelle Situation erzwingen würden. Man könnte von einer Art Irrealis der Unterstellung sprechen: Wenn das damals so gelaufen wäre, wie xy das behaupten, dann müsste das heute so aussehen bzw. müsste heute eine bestimmte Situation bestehen. Man unterstellt also einen bestimmten gegenwärtigen Zustand als Ergebnis eines Geschehens, dessen Nichtexistenz das vergangenen Geschehen (mindestens in der behaupteten Form) als unmöglich, also unwahr, erweist. Wie bei dem chronologischen Argumentieren herrscht hier der Satz des Widerspruchs.

Zugleich gibt es hier eine besondere Verschränkung von Vergangenheit und Gegenwart: Im ersten Falle ragen die vergangenen Dinge noch in die Gegenwart hinein. Herodot spricht dann von es eme eti („noch bis auf meine Zeit“) und gebraucht gerne das durative Imperfekt (Hdt. 5,45,2). Es ist mithin noch etwas Sichtbares da, es gibt also eine Beziehung zum wertvollen Erkenntnisorgan des Sehens. Im zweiten Falle werden vergangene Ereignisse mit einer gegenwärtigen Konstellation gedanklich-experimentell verbunden: Auch hier hat man etwas vor Augen, das als Kriterium dient (es müsste eben anders sein, wenn eine bestimm-

44 Gehrke 1998, 185-188 (mit weiteren Hinweisen), vgl. jetzt auch Bichler 2013, 26-28.

45 Herodot 5,45 - ich verdanke die folgenden Beobachtungen der Lektüre von Texten Elisabetta Lupis. 
tes Geschehen in der Vergangenheit eingetreten wäre) - nur kommt es hier deutlich auf das Nach-Denken und die Urteilsfähigkeit, die gnōmē, an.

Die hier begegnenden Standards sind bei Herodot bereits vorgefunden. Die Beobachtungen und Argumente wurden ja ganz offenkundig von den Repräsentanten der hier beteiligten Poleis - in dem einen Falle sogar einer physisch zerstörten - geäußert, von Politikern also und / oder von Personen, die dort gleichsam für die Geschichte verantwortlich waren. Dies war nicht zwingend eine offizielle oder gar schriftlich fixierte Überlieferung, aber doch so etwas wie eine „lebendige Tradition“ (Giangiulio 1989, 192). Unverkennbar ist die strukturelle Nähe solcher Argumentationen zur Forensik. ${ }^{46}$ Auch dort kam es ja darauf an, ein häufig nur durch Zeugen, also durch Hörensagen ( $a k o \bar{e})$ zugängliches Geschehen wahrheitsgemäß (also adäquat dem Geschehen) zu ermitteln - oder in den Gerichtsreden argumentativ eine solche Wahrheit zu präsentieren. Es ist charakteristisch, dass Herodot, also die kritische Recherche-Historiographie, diese Grundsätze teilt und intellektuell verfeinert. Die entscheidenden Momente waren klar. Nino Luraghi $(2001,143)$ hat sie so formuliert: „personal experience and reasoning are stronger arguments than ,what people say““.

Dass Herodot mit diesen Grundsätzen kein Einzelfall blieb, lag nicht nur an seiner Wirkung, sondern auch daran, dass er im Sinne der erwähnten Hypolepse, ähnlich wie er sich seinerseits schon auf Hekataios bezog, also durchaus kompetitiv, einen nachahmenden Konkurrenten fand. Dieser schritt auf dem gebahnten Wege energisch und mit besonderer Konsequenz voran, hat aber gerade damit Herodot weitergeführt und transzendiert. ${ }^{47}$ Er verstärkte das Moment des kritischen Prüfens und des intellektuellen elenchos gerade im Hinblick auf das Ermitteln und die Genauigkeit, die Wahrheit, wie er sie verstand, mit einer besonderen Skepsis gegenüber der Überlieferung und den traditionellen Formen ihrer Pflege, insbesondere wenn sie sich ins Gewand der Rhetorik kleidete, wie Jonas Grethlein jüngst gezeigt hat. ${ }^{48}$ Er reizte das Prinzip besonders aus, indem er die ältere Geschichte geradezu nach den Regeln der Logik - einer anthropologischen Machtlogik - behandelte, womit er fast zwangsläufig Eigenoptik und Zeitgeschichte als Ausgangspunkt nahm: Diese war nicht nur (in Gestalt des Pelopon-

46 Man denke an die Bemerkungen zur Elenktik, o. S. 73. In diesem Rahmen sind auch die Bemerkungen von Nagy 1988 zum Zusammenhang von Operationen der historiē mit Schiedsgerichtsverfahren zu berücksichtigen.

47 S. besonders Corcella 2006, 51, vgl. Rogkotis 2006 („creative continuation, development, and refinement“, 86) und Schepens 2007, 47; zur Nähe zwischen den beiden Historikern s. auch Foster/Lateiner 2012, 1-9.

48 Vor allem Grethlein 2005. 2010, 220-240. 
nesischen Krieges) der eigentliche Gegenstand, sondern sie prägte auch mit den aus ihr abgeleiteten Prinzipien die Rekonstruktion der älteren Geschichte. ${ }^{49}$

Damit bildete Thukydides definitiv ein Modell, wie schon daraus erhellt, dass mehrere Historiker direkt an ihn anschlossen und sein Werk schlicht fortsetzten, darunter bekanntlich Xenophon. Wenn man hinzunimmt, dass Thukydides selber in seiner Darstellung der Pentekontaetie, ${ }^{50}$ wenn auch thematisch orientiert, den zeitlichen Anschluss an Herodot findet, dann ergibt sich hieraus gleichsam eine fortgeschriebene griechische Geschichte bzw. ein fortlaufender Bericht über die „griechischen Angelegenheiten“ (ta hellēnika). Das blieb im jeweiligen zeitlichen, sozusagen biologischen Rahmen der Autoren und Generationen, aber letztlich immer in deren jeweiliger Gegenwart - wobei das kritische Potential durchaus variierte und sich auch markante Veränderungen ergaben.

Die Historiographie - so wie sie entstanden war und sich in den ersten drei Generationen von Herodot über Thukydides $\mathrm{zu}$ Xenophon und anderen entwickelt hatte - benötigte in diesem Sinne eigentlich keine Vergegenwärtigung, weil sie im Kern präsentisch war. Man kann im übrigen von hier aus innerhalb der gesamten griechischen Historiographie, eigentlich bis in die spätbyzantinische Zeit, eine fortlaufende Linie von Autoren ziehen, die immer wieder aneinander anknüpften, nicht ohne Polemik zwar, aber doch so die griechische Geschichte insgesamt von der Perspektive eigenen Erlebens und der eigenen Lebensspanne her stetig fortschreibend. ${ }^{51}$

Wie sollte man aber nun (wenn man nicht, wie Xenophon - die Kyrupädie liegt auf einer anderen Ebene -, völlig darauf verzichtete) mit der entfernteren Vergangenheit umgehen, die man ja schließlich aus den erwähnten Epen, Gedichten, Erzählungen, Reden und Bildern in ihrer ganzen Vielfalt und Varianz kannte, Bildern und Geschichten, von denen man geradezu überflutet wurde, beim Fest und Kult, in der Volksversammlung und sogar vor Gericht? Herodot wollte die Dinge, wie wir sahen, schlicht festhalten, auch wo er sie nicht erkenntnistheoretisch durchdringen konnte, und tendierte gerade unter dem Aspekt der Lehren, die man aus der Geschichte ziehen sollte und konnte, für eine größere zeitliche Tiefe.

$49 \mathrm{Zu}$ Thukydides' ,Archäologie‘ in diesem Sinne s. Gehrke 1993, vgl. auch Tsakmakis 1995, 25-63 und Nicolai 2001.

$50 \mathrm{Zu}$ deren narrativer Struktur vgl. jetzt Gehrke 2013c.

51 Hierzu s. besonders Canfora 1971/1999/2011 und Fornara 1983, 46; wie auch immer man das gattungsmäßig differenzieren mag (hierzu jetzt Tuplin 2007), das Bedürfnis, zeitnah zu berichten und an Vorgänger anzuknüpfen (bzw. an in zeitlicher Nähe zu ihnen angesiedelte historische Zäsuren), war offensichtlich. 
Demgegenüber dehnte Thukydides, wie schon angedeutet, das rationale Verfahren und die kritische Prüfung noch weiter auf die frühe Vergangenheit aus, wohl wissend - wie am Beispiel der Tyrannentöter sichtbar gemacht wird -, dass dies noch schwieriger war als die Recherche unter Zeitzeugen. Zugleich dokumentiert er in dem erwähnten Exkurs über die attischen Tyrannentöter in praxi, wie man sich auch zu dem Älteren, dem echt Vergangenen, einen Zugang bahnen konnte, nämlich im wesentlichen mittels Forschung und elenchos: mit einer Musterung von Dokumenten und Relikten sowie dem Verweis auf zuverlässige Quellen, aber nicht zuletzt auch mit rationalen - auch common-sense-Argumentationen aufgreifenden - Operationen, Schlussfolgerungen und Widerlegungen (etwa zur Struktur der Tyrannis als Alleinherrschaft und zum Alter der Tyrannen). Das ähnelt in vielem Herodots methodischem Vorgehen (man denke an das vorhin erwähnte Beispiel zu Sybaris und Kroton ${ }^{52}$ ) und steht ebenfalls dem Forensischen nahe.

Mit solchem Blick auf das Präteritum unterzog Thukydides nicht nur die ältere Überlieferung, sogar eine Autorität wie Homer, einer kritischen Überprüfung, sondern entwickelte darüber hinaus auch ein gedanklich-logisches Instrumentarium zur Rekonstruktion auch der weiter zurück liegenden Vergangenheit, das er aus Beobachtungen und Reflexionen des politischen Geschehens, vor allem des von ihm selbst erfahrenen und bedachten, abstrahierte. Zwar macht er das vor allem in einem Abschnitt zu Beginn seines Werkes, der so genannten Archäologie, ${ }^{53}$ deutlich, der stark argumentativ geprägt ist, weil es ihm nicht um die Alte Geschichte schlechthin ging, sondern um den Beweis darum, dass ,sein“ Krieg der größte bisher da gewesene war. Aber die frühe Zeit kommt damit in den Blick, und Thukydides zeigt, wie er mit ihr umgeht, und dass dabei bestimmte Regeln maßgeblich sind. Gerade diese waren ihm aber - das zeigt die Rede vom ktēma es aei $(1,22,4)$ - besonders wichtig, denn es handelte sich um Gesetzmäßigkeiten, die sich aus der im wesentlichen gleich bleibenden Natur des Menschen ergeben.

Dieser Blick auf die Vergangenheit kann durchaus als eine Vergegenwärtigung verstanden werden. Bei ihm haben ja gerade Zeitanalysen und - erfahrungen eine Rolle gespielt, freilich auch philosophische Reflexionen über die menschliche Natur, die ihrerseits auf ältere Weisheiten und Einsichten, auch und gerade solche der Poesie, zurückgreifen konnten. Wir können das besonders in der seinerzeitigen Tragödie beobachten, und manche Entsprechung zwischen Thukydides und Euripides unterstreicht dies. Insofern ist übrigens die bekannte Differenzierung in der Poetik des Aristoteles zu relativieren. ${ }^{54}$

52 Aber etwa auch an die common-sense-Argumentation bei Herodot $(2,120)$ zu Helena.

53 Thukydides 1,1,2-1,21,2; vgl. o. Anm. 49 und u. Anm. 56.

54 Vgl. auch Erler 1997, $93 \mathrm{zu}$ „Generalisierungen“ bei Thukydides. 
Die Vergangenheit gerät also nicht nur in den Blick des aufmerksam-kritisch ermittelnden Historikers, sondern auch einer historisch-philosophisch reflektierten und reflektierenden Analyse allgemein menschlichen Verhaltens, die mit eigenen Zeiterfahrungen rückgekoppelt und abgeglichen wird. Geschichte auf diese Weise, mit dem Anspruch auf Wahrheit und Wirklichkeit, neu zu konstituieren ist ein überaus kühner Gedanke, in dem Thukydides, wenn ich richtig sehe, keinen wirklichen Nachfolger fand. Auch bei ihm liefert also die Gegenwart den Ausgangspunkt. Sie wird aber nicht lediglich zurückprojiziert, sondern aus Logik und nach Regeln, die überzeitlich gelten, gleichsam in die Vergangenheit zurückgeschrieben. Sie ist dann für nachdenkende Menschen gut verständlich und nachvollziehbar, insofern auch nah und gegenwärtig, wenn man so will. Geschichte wird hier nicht schlicht weitergegeben, sondern gleichsam produziert, von einer Gegenwart her, aber nicht im Sinne erklärender und erbaulicher Erzählungen, sondern nach einer Logik des Geschehens gerade mit dem Blick auf dessen zeitlose Elemente.

Was aber geschieht nun mit der ,richtigen‘ älteren Geschichte, wie die meisten Griechen sie sahen, mit den alten Erzählungen, von dem, was blieb, was berichtet wurde. Thukydides griff ja nur auf, was er argumentativ brauchte, im Sinne der auxēsis am Anfang des Werkes ${ }^{55}$ bzw. zur Illustrierung der Größe und Bedeutung Siziliens im 6. Buch oder in der Polemik gegen die falschen Geschichtsvorstellungen der Athener. Wie aber werden die alten Geschichten und Erzählungen ansonsten in der griechischen Historiographie vergegenwärtigt? Auch hier liefert Herodot die Beispiele: Es ist - sozusagen ganz traditionell - der Modus des Erzählens, genauer: der narrativen Vergegenwärtigung, welche durch das Operieren mit den eingelegten Reden noch verstärkt wird. ${ }^{56}$ Immer wieder wird neu und anders erzählt, die Vielfalt der Varianten und der Performanz hält das Alte lebendig, in der wachsenden Vielfalt der Geschichtsschreibung mit ihrer eigenen Agonalität nicht anders als in der von Varianten strotzenden Literatur und Kunst. ${ }^{57}$

Hier hat sich dann auch eine zunehmende Interaktion zwischen der - sagen wir - populären Geschichte in der Kunst und der Rhetorik und der intellektuellen Historiographie ergeben, bei aller Polemik von Seiten letzterer. Diese betraf nicht nur den Gegenwartsbezug und die Zeitgeschichte, sondern auch die langen

55 Maddoli 1994, vgl. Luraghi 2000.

56 Zur Vermittlung von Vergangenheit und Gegenwart in den Reden s. Marincola 2007, 130. Zu den Reden in der antiken Historiographie generell vgl. jetzt den Überblick bei Achilli 2012.

57 Aspekte der Narratologie werden in der neueren altertumswissenschaftlichen Forschung immer stärker in den Blick genommen, vgl. etwa, exempli gratia, die Beiträge in Grethlein/ Rengakos 2009 sowie bei Foster/Lateiner 2012. 
Linien, die sich in die Vergangenheit zurückziehen ließen, immer in Verbindung mit der Gegenwart. Es blieb stets bei der traditionellen Verlebendigung und damit Vergegenwärtigung der Geschichte in Monument und Fest. Und immer mehr entfaltete die Rhetorik - ohnehin mehr und mehr das Medium stilistisch-sprachlicher Formung - ihre Vergangenheitsbilder vor allem im Licht epideiktischen und insofern gegenwärtigen Rühmens.

Hier gab es immer auch Bezüge auf die jeweiligen Träger der Geschichte, auf die sozialen Gruppen und die politischen Gemeinschaften, die in diesem Rahmen ihre intentionale Geschichte formten und weiter ausgestalteten. Im Athen des 5. Jahrhunderts sind es gerade Tragödien sowie die aus Anlass der PanathenäenFeste und der Staatsbegräbnisse gehaltenen Reden, die hier zu nennen sind und die man als Medien durchaus in einem Zusammenhang sah. ${ }^{58}$ Man hat geradezu, wie schon erwähnt, von einer „Erfindung Athens“ (Nicole Loraux) gesprochen, und dazu gehörte nicht zuletzt ein historisches Selbstbild, das die Athener der Vergangenheit und der Gegenwart in einer gradlinigen Entwicklung als Beschützer der Schwachen und als Vorkämpfer hellenischer Freiheit gegen die barbarischen Angreifer sahen. Sie hatten das in der entfernten - mythistorischen Vergangenheit gezeigt, gegen die Amazonen und die Thraker des Eumolpos, für die Argiver nach dem Zug der Sieben und für die Herakliden, und besonders in der jüngeren und noch ganz nahen Vergangenheit, in den Kriegen gegen die Perser. Und so würden sie dann auch in der Zukunft sein. ${ }^{59}$

Generell aber hat sich nicht zuletzt der Siegeszug der Rhetorik im 5. Jahrhundert nachhaltig ausgewirkt, nicht nur auf die intentionale Geschichte der Griechen, sondern auch auf die zunächst neue Form der intellektuell-elenktischen Geschichtsschreibung. Das sollte sich dann im 4. Jahrhundert schnell zeigen. Aber schon um 400 v. Chr. sind verbreitete Vorstellungen geschichtlicher Abläufe im Sinne einer intentionalen Geschichte der Griechen, ja der Welt in einem rhetorisch-philosophischen Traktat greifbar, in den dissoi logoi (,Zweierlei Ansichten“). Dort wird im Zuge einer fundamentalen moralischen Debatte - es geht um nichts weniger als um das Gute und Schlechte (agathon und kakon) - ein historisches Argument ausgespielt (1,8-10). Analog zu den erwähnten historischen Linien der Athener kann der Autor dabei in einer Reihe zurück gehen, vom ganz Aktuellen bis fast zu den Anfängen: vom Peloponnesischen Krieg über die Perserkriege, den Troischen Krieg, den Kampf der Sieben gegen Theben, den Konflikt der Lapithen und Kentauren bis hin zur Gigantomachie. Gerade diese

58 Gorgias B 11, 9 Diels/Kranz; vgl. auch die Fallstudien von Grethlein 2010.

59 Loraux 1981/1993; Gehrke 2003, 20-23; zur intentionalen Geschichte der Athener in diesem Sinne, mit einer Fokussierung auf die Thebaner, s. Steinbock 2013. 
argumentative Verwendung von Geschichte zeigt die Geläufigkeit der Vorstellungen, sie gibt mithin einen klaren Blick auf die Geschichte aus Sicht der Griechen in einem groben Überblick: Es geht um ein weithin geteiltes Wissen um eine Vergangenheit, die mit der Gegenwart verbunden und verschränkt ist und vice versa.

Aber wie steht es um die Wahrheit, um die Größe, die ja gerade in dem Neuansatz der intellektuellen Historiographie so zentral war? Hier war der Sieg der Rhetorik fatal, man ist versucht, von einem Pyrrhussieg zu sprechen. Denn wodurch wurde nun die Wahrheit verbürgt? Zunächst wiederum durch die intellektuelle Debatte und den Kampf der Widerlegungen. Auch und gerade in der Sophistik und damit in der Rhetorik, die sich nun als lehr- und lernbare Kunst etablierte, war die Frage nach der Wahrheit ein wesentliches Thema. ${ }^{60}$ Einer der ganz großen Meister dieser Kunst, Gorgias von Leontinoi, propagiert sogar buchstäblich die Orientierung der epideiktischen Rede, der Eloge, auf das Wahre, das auf kritischer Prüfung beruht. Aber dazu gibt es auch eine andere Seite, die der große Meister selbst in dem eigens verfassten Prunkstück vorführt, das sein Exempel bildet, in der Lobrede auf die schöne Helena. Die Redner, ja Autoren schlechthin (und damit auch Geschichtsschreiber) können auch, wenn sie auf Bezirzen und Berücken orientiert sind wie die Dichter, eine „trügerische Rede erfinden“ (pseudē logon plasantes) und damit „erfreuen und überzeugen“, mit einer nicht an der Wahrheit orientierten Kunstfertigkeit. ${ }^{61}$ Dass der Rhetor am Schluss dann das, was er da so bezaubernd vorträgt, als „Spielerei“ (paignion) bezeichnet (B11, 21), macht es auch nicht gerade besser. Das Trügerische, das dem Wahren ähnlich ist - genau hier ist es wieder, nun auch noch im modernen Gewand der Rhetorik. Auch und gerade hier konnte man wieder ins Grübeln kommen. Der Kampf um die Wahrheit war noch nicht erledigt. Wie er sich in der griechischen Historiographie fortsetzte (wiederum im Spannungsfeld von Vergangenheit und Gegenwart) und was wir gegebenenfalls daraus lernen können, werde ich im folgenden Kapitel abschließend zu zeigen versuchen.

60 Vgl, hierzu auch Gehrke 1993, 14.

61 Gorgias 82 B 11, 1f.10f.13 Diels/Kranz. 